\title{
Research on Rapid Dynamic Rendering and Modeling Technology of Water Landscape Based on 3D Image Technology
}

\author{
Li Sun (iD \\ School of Art, Anhui University of Finance \& Economics, Bengbu, Anhui 233000, China \\ Correspondence should be addressed to Li Sun; 120081141@aufe.edu.cn
}

Received 17 December 2021; Revised 12 January 2022; Accepted 17 January 2022; Published 28 February 2022

Academic Editor: Baiyuan Ding

Copyright (C) $2022 \mathrm{Li}$ Sun. This is an open access article distributed under the Creative Commons Attribution License, which permits unrestricted use, distribution, and reproduction in any medium, provided the original work is properly cited.

\begin{abstract}
With the development of computer graphics technology, the application of 3D visualization simulation technology has become increasingly widespread. This paper develops a three-dimensional visualization simulation system for water bodies by studying the general model of regional landscape space and water quality purification. This paper mainly does research on rapid dynamic rendering and modeling technology of water landscape based on 3D image technology and uses the 3ds Max Material Editor to map the enhanced 3D landscape design to complete the design, and the designed landscape feature data will be saved in the system data statistics unit for later query. This article also expounds the entire process of alternative simulation construction and confirms the feasibility and superiority of alternative modeling methods through multiple case comparisons. Alternative modeling methods are used as the main way to construct waterfall scenes, that is, simple models are combined with texture mapping, particle effects, and other forms to replace complex models, while ensuring the simulation effect, and the complex models are presented in the most simplified way to make the virtual scene. It is well performed in terms of authenticity and real time. The research results show that the designed system design renderings are extremely clear and of good quality; they have a high score in the water landscape design. Moreover, the system application cost is low, energy consumption is low, and operation efficiency is high.
\end{abstract}

\section{Introduction}

Water body is a common natural scene in real life. Simulation research on water body has important theoretical significance and practical value in many fields such as landscape simulation, online games, film and television special effects, and water conservancy engineering. Due to the irregular shape and random variability of water bodies, water body simulation has become a research difficulty and hotspot in the simulation watershed. At the same time, it has also aroused the interest of countless researchers. The water body is simulated. However, due to the limitations of computer hardware equipment, there is still a contradiction between the authenticity and real-time nature of water simulation. Fish and bear's paw cannot be achieved at the same time. This requires a balance between authenticity and real time [1-5]. The 3D image technology is shown in Figure 1.

The application field of water body simulation requires simulation effects so that water body simulation has three characteristics: authenticity, real time, and interactivity. Authenticity refers not only to similarity in form, but also to having a sense of reality in terms of object features. At present, particle systems and fluid dynamics are mainly used to express the shape of water bodies, and rendering techniques such as environment mapping and ray tracing are used to realize the refraction of light by water bodies. In terms of real-time performance, technologies such as GPU hardware acceleration and LOD are used to increase the rendering speed and ensure the real-time performance of water body simulation. Interactivity refers to the dynamic effects of the interaction between the water body and other objects, such as waves caused by the ship when sailing and floods hitting buildings. Authenticity and real time are the goals that water simulation research and development are constantly pursuing, but these two characteristics have very high requirements on computer hardware. When pursuing high real-time effects, realism is often sacrificed. Water simulation modeling methods are controlled by the 


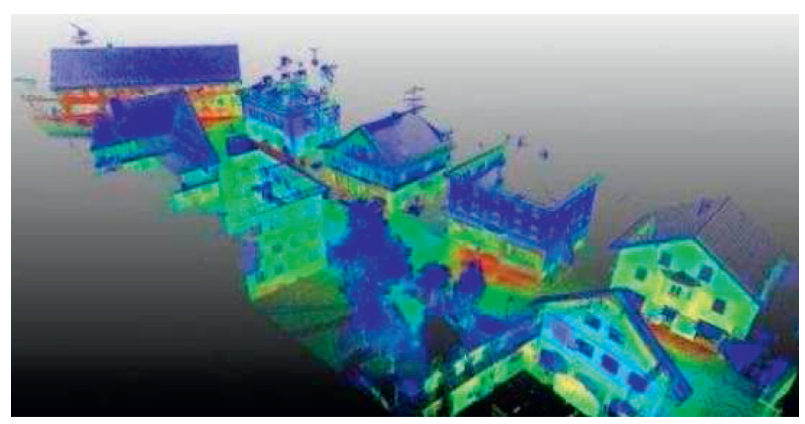

Figure 1: 3D image technology.

development of computer software and hardware. In the early days, texture mapping and parameter modeling were generally used to achieve simulation effects. The development of GPU technology enabled the use of particle systems, fluid dynamics, and other methods. The authenticity of water simulation effects, real time, and interactivity are also gradually increasing. At present, the common water simulation performance methods include texture mapping, surface parameters, particle systems, fluid dynamics, and wave functions. With the continuous improvement of water body simulation requirements, modeling methods tend to be integrated with multiple methods, and comprehensive treatment is adopted to complement them in order to achieve better water body simulation results [6-12].

Compared with other landscapes, water landscapes are more distinctive. They not only meet the objective requirements of human aesthetics, but also have aesthetics and spiritual contributions. Water landscape is the product of the combination of human aesthetic standards, culture, and water [13-18]. It is the evolution product of human civilization that perfectly integrates human mythology, literature, and other conceptual forms and tangible entities such as architecture and ornaments with the water landscape of a certain designated area. People use the principle of VISC conversion of computer graphics to realize the conversion of data to graphics, display the graphics on the screen, and finally meet their needs through interactive processing. From a long-term perspective, people will still meet their different needs from different data structures, algorithms, and mathematical theories, and they will also get a variety of three-dimensional visual models according to different computer software and hardware configurations. These calculated accurate models will be put into actual production. Regarding the actual requirements for the visualization of water bodies in the water system, the water quality model can be used to simulate the required water bodies [19-21].

Traditional methods usually use virtual design methods to improve the authenticity of water design works. However, the virtual design method has disadvantages such as large data collection error and picture distortion. With the rapid development of electronic technology, electronic drawing and computer visualization technology are gradually optimized. Since the 1960s, two-dimensional drawing has evolved into the current three-dimensional virtual simulation method [22-26]. The three-dimensional virtual simulation method can present the actual environment with high precision. It has important application value in the fields of medicine, urban planning, design, and manufacturing. Therefore, this article applies 3D image processing technology to landscape design, designing a landscape design system based on 3D image processing technology [27-33].

\section{Water Landscape Design System Based on 3D Image Processing Technology}

2.1. Hardware Design. Virtual reality technology is a computer-generated three-dimensional digital model of a scene model that exists or does not exist in reality and imports the virtual reality technology into the virtual reality software to generate a visual, auditory, and tactile model that can be viewed from all angles. The modeling technology of water landscape based on 3D image technology is shown in Figure 2, which is mainly used to store garden design examples, plant picture collections, plant graphic symbol libraries, and scenery picture collections, which are used to provide designers with landscape design materials. In the landscape planning of the model scene design unit, the scene design is mainly completed through the three-dimensional model. The structure of the three-dimensional model is shown in Figure 3. This module mainly contains the architectural landscape point elements, which are directly produced using ArcGIS 10.2. First, construct a three-dimensional geometric model, and introduce the basic building information and the two-dimensional CAD distribution map. Set real textures to the buildings to achieve a landscape design close to reality. The 3D landscape image can be preprocessed during design. Finally, the $3 \mathrm{ds}$ MAX material editor is used to the enhanced 3D landscape design drawing for texture mapping. The feature data such as landscape name, basic functions, land area, and elevation data are all optimized with ArcMap to construct a feature database for landscape design.

2.2. 3D Image Processing. The rendering of model colors in landscape 3D design must be implemented based on real landscape images. Decompose the covariance of $L_{w}$ according to the relevant theory:

$$
D_{L_{w}}=F\left[L_{w}^{T}\right]=\mathrm{FEF}^{T},
$$

where $E$ represents the diagonal matrix of eigenvalues and $F$ represents the orthogonal matrix. Then, the linear whitening matrix is

$$
U=\frac{F^{T}}{\sqrt{E}} .
$$

Through the above analysis, $s$ covariance matrices can be obtained:

$$
\begin{aligned}
D_{s} & =F\left\{s s^{T}\right\} \\
& =U F\left\{L_{w} L_{w}^{T}\right\} U^{T} .
\end{aligned}
$$

In order to overcome the shortcoming, that is, the $3 \mathrm{D}$ image base is too difficult to roughly increase the computational difficulty, the $3 \mathrm{D}$ image features must be processed by dimensionality reduction. Distribute the eigenvalues in 


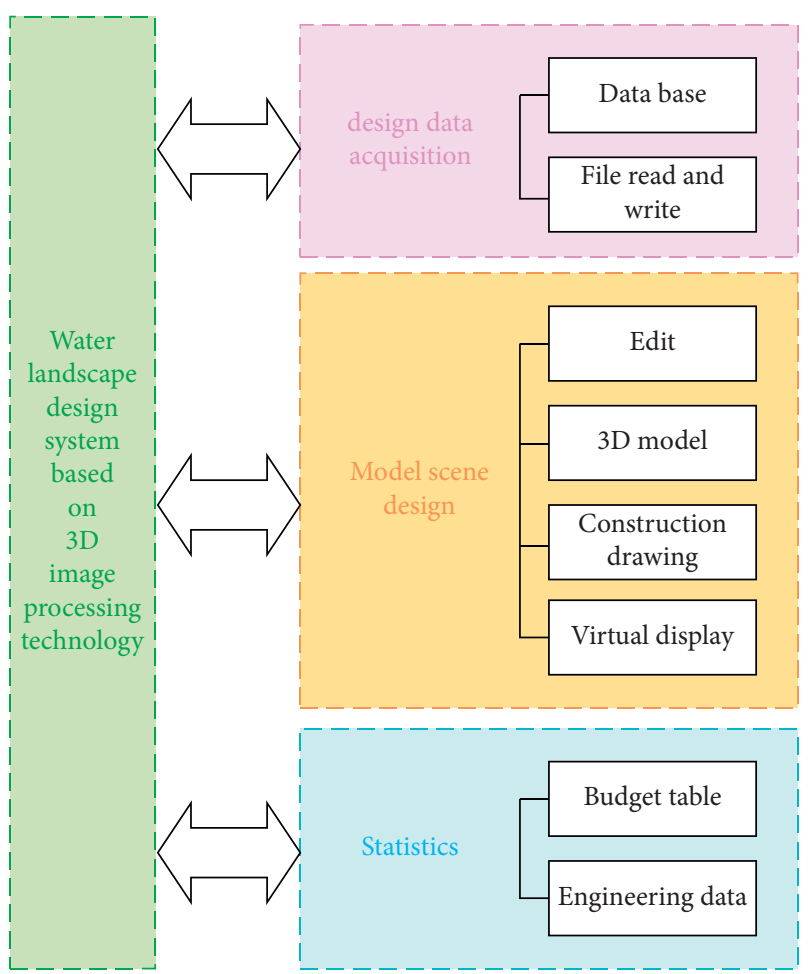

Figure 2: The landscape design system structure of 3D image processing technology.

descending order, and select eigenvalues with large values to obtain the reduced-dimensional diagonal matrix; then,

$$
U=\frac{1}{\sqrt{E} D^{T}}
$$

where $D$ describes the variance and $U$ represents the $3 \mathrm{D}$ landscape image after dimensionality reduction. After the above operations, the observation vector of the $3 \mathrm{D}$ landscape image is

$$
s(t)=\left[s_{1}(t), s_{2}(t), \ldots, s_{a}(t)\right]^{T} .
$$

The above formula conforms to

$$
s(t)=\widehat{U} L_{w}(t) .
$$

Then, the statistics of the independent components of the $3 \mathrm{D}$ landscape image are

$$
z_{j}(t)=\left[\beta_{j 1}, \beta_{j 2} \ldots, \beta_{j a}\right]\left[\begin{array}{c}
s_{1}(t) \\
s_{2}(t) \\
\ldots \\
s_{a}(t)
\end{array}\right] .
$$

In the formula, $\beta \mathrm{ja}$ describes the independent components of the $3 \mathrm{D}$ landscape image. In summary, selecting a reasonable image base for 3D landscape images can minimize the amount of calculation while not causing 3D image distortion. The auxiliary virtual environment gives people a sense of psychological reality and can directly act on all objects in this virtual environment. The human-computer

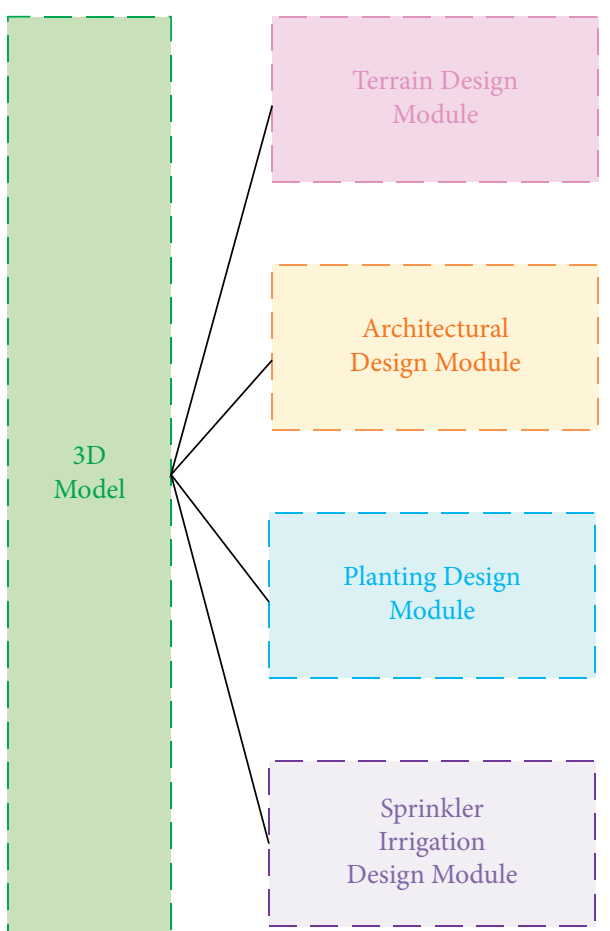

Figure 3: The structure of the three-dimensional model.

interaction functions of virtual reality technology provide people with a platform for experience and simulation training anytime and anywhere. First of all, it plays a pivotal role in simulation training in the military and aerospace fields. The evaluated $x$ and $y$ are shown in Figure 4 .

After the 3D landscape image is preprocessed, when enhancing the 3D landscape image, the 3D landscape image is first enhanced in the frequency domain, and the pixel grayscale of the image is converted into a new image grayscale based on a fixed function to obtain the image grayscale feature. Obtain the enhancement function of the gray contrast of the edge of the image, and realize the enhancement of the 3D landscape image features. Suppose $g^{\prime \prime}(a, b)$ is used to describe the preprocessed 3D landscape image, $y_{\text {jui }}^{\prime}$ describes the neighborhood of random pixels $d_{s g}^{\prime}$ in the $3 \mathrm{D}$ landscape image, $v_{\text {wer }}^{\prime}$ describes the number of values of each image feature, and $c_{\text {wer }}$ ' describes the total value of the number of image pixels; then, use the following equation to enhance the image in the frequency domain:

$$
K_{\mathrm{qwu}}^{\prime}=\frac{c_{\text {wep }}{ }^{\prime} g^{\prime \prime}(a, b)}{y_{\text {jui }}^{\prime} d_{s g}^{\prime}}\left\{v_{\text {wer }}^{\prime} \oplus h_{\text {gtu }}{ }^{\prime}\right\} .
$$

In the formula, $h_{\mathrm{gtu}}{ }^{\prime}$ describes the gray value distribution of the 3D landscape image. Assuming that the number of occurrences of various gray values in a $3 \mathrm{D}$ landscape image is set to $\theta_{(a, b)}{ }^{\prime}$ which describes a random point in the image, $(a+n, b+m)$ describes the disturbance point of the image, and $(a+n, b+m)^{k l}$ describes the corresponding points of $(a$, $b)$ and $(a+n, b+m)$; then, obtain the new image grayscale by the following formula: 


$$
c_{\text {poi }}^{\prime}=\frac{(a+n, b+m)^{k l}}{(a, b)(a+n, b+m)} \oplus \frac{\theta^{\prime}}{y_{\text {jui }}^{\prime}} .
$$

Assuming that the first-order differential function of the image is $\vartheta_{\text {poi }}{ }^{\prime}$, the inherent characteristic of the $3 \mathrm{D}$ landscape image is $u_{\text {wer }}^{\prime}$, and the amplitude-frequency characteristic function is set to $e_{\mathrm{sgh}}{ }^{\prime}$; then, the grayscale characteristics of the image can be obtained by the following formula:

$$
r_{\text {yup }}^{\prime}=\frac{e_{\text {sgh }}^{\prime} \pm \vartheta_{\text {poi }}^{\prime}}{u_{\text {wer }}^{\prime}} \pm e_{\text {sgh }}^{\prime} .
$$

It is assumed that the variance of the original image block and each adjacent basic image block after merging is $\varphi_{\text {uip }}{ }^{\prime}$, and the ratio of the total image occupied by each image block is set to $\lambda_{\text {wepp }}$. The enhancement function of the edge gray contrast of the $3 \mathrm{D}$ landscape image is

$$
\varepsilon^{\prime}=\frac{\lambda_{\text {wepp }}{ }^{\prime} \varphi_{\text {uip }}{ }^{\prime}}{g_{t u}^{\prime}} g_{\text {rty }}^{\prime} .
$$

In the formula, $g_{t u}{ }^{\prime}$ is the mask operator and $g_{\text {rty }}{ }^{\prime}$ is the texture attribute in the low frequency range. The predicted value is shown in Figure 5.

Assume that the texture attribute weight space in the low frequency range is $\eta$ and that the relevance of each pixel in the 3D landscape image signal when it is near in the spatial domain is set to $M_{\mathrm{POL}}{ }^{\prime}$; then, the $3 \mathrm{D}$ landscape image feature enhancement method is

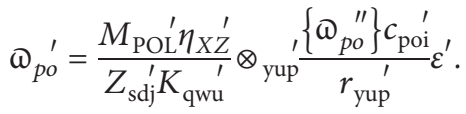

In the formula, $Z_{\text {sdj }}{ }^{\prime}$ describes the structural information of the 3D landscape image and $\omega_{p o}$ " describes the variance of the original image block and the basic image block of each neighbor. Based on the above processing, the 3D landscape image feature enhancement can be achieved, and the landscape design image clarity is improved.

\section{3D Visualization Simulation Technology}

In recent years, with the continuous improvement of virtual reality technology and the rapid advancement of computer technology, people have become more and more aware of the importance of virtual reality technology, and it has been used in many industries, showing broad development prospects. Developed countries began to study the emerging research field of scientific visualization in the late 1980s. It can effectively obtain accurate data. The essence is to track the simulation calculation process through graphics and image processing, and the results are on the screen. The above shows that the interactive processing method closely integrates the graphics and image processing understanding technology and the human-computer interaction technology and finally produces complex multidimensional data image graphics. At present, the field of scientific computing visualization research mainly focuses on the research of computing environment, the research of virtual environment display equipment, and the research of scientific computing visualization technology. (1) As for research on the computing environment, in the high-end computing environment, massively parallel computers with distributed storage, symmetric multiprocessors with shared internal storage, and multiprocessors with distributed shared internal storage have come out one after another. In low-end aspects such as PCs, multiprocessor structures and parallel processing functions have also appeared to improve the quality and efficiency of scientific computing. (2) The research of virtual environment display devices and the development of wall-type large-screen displays are suitable for true three-dimensional display. The cave-like display device with immersive characteristics provides methods and means for the generation and performance of huge amounts of data. (3) There are currently two main types of scientific computing visualization technology that can be studied: one is the study of various application models, and the other is visualization technology. The research of various application models is based on simulation and design application models. It uses visual insight to interactively solve various problems and processes data from different disciplines such as medicine, geology, meteorology, physics, and biological sciences. Graphical information that can be intuitively accepted by human vision and the research of visualization technology, distributed, collaborative, and immersive technologies have become the current mainstream research directions. The prediction is compared in Figure 6.

Water simulation is an important research and development direction of virtual reality technology. In a virtual reality scene, in order to increase the richness and realism of the scene, it is often necessary to add a large number of natural landscapes. Focusing on the needs of the water purification system and in accordance with the requirements of project construction, the system can be roughly divided into three parts: a water purification module, a visual simulation module, and a data management platform. The water purification module includes four sub-function modules: water purification model parameter management, model establishment, model calculation, and result query. The visual simulation module includes six sub-function modules: scene management, modeling, channelization, topography, geophysicalization, and water quality. The data management platform includes six sub-function modules: database parameter management, module data management, channel data management, terrain data management, surface data management, and spatial data management.

1. The water purification module is one of the core modules implemented in this project. It is based on channel modeling, using relevant spatial information and model data provided by the data management platform to dynamically establish a water purification model, calculate and analyze the water quality based on system parameters setup, and then form a result query record to provide guiding information for decision making. 2. The visual simulation module is another core module of this project. It is based on the data management platform to extract channel data, terrain data, feature data, and related data information; use OpenGL technology to dynamically draw channels, terrain, features, 

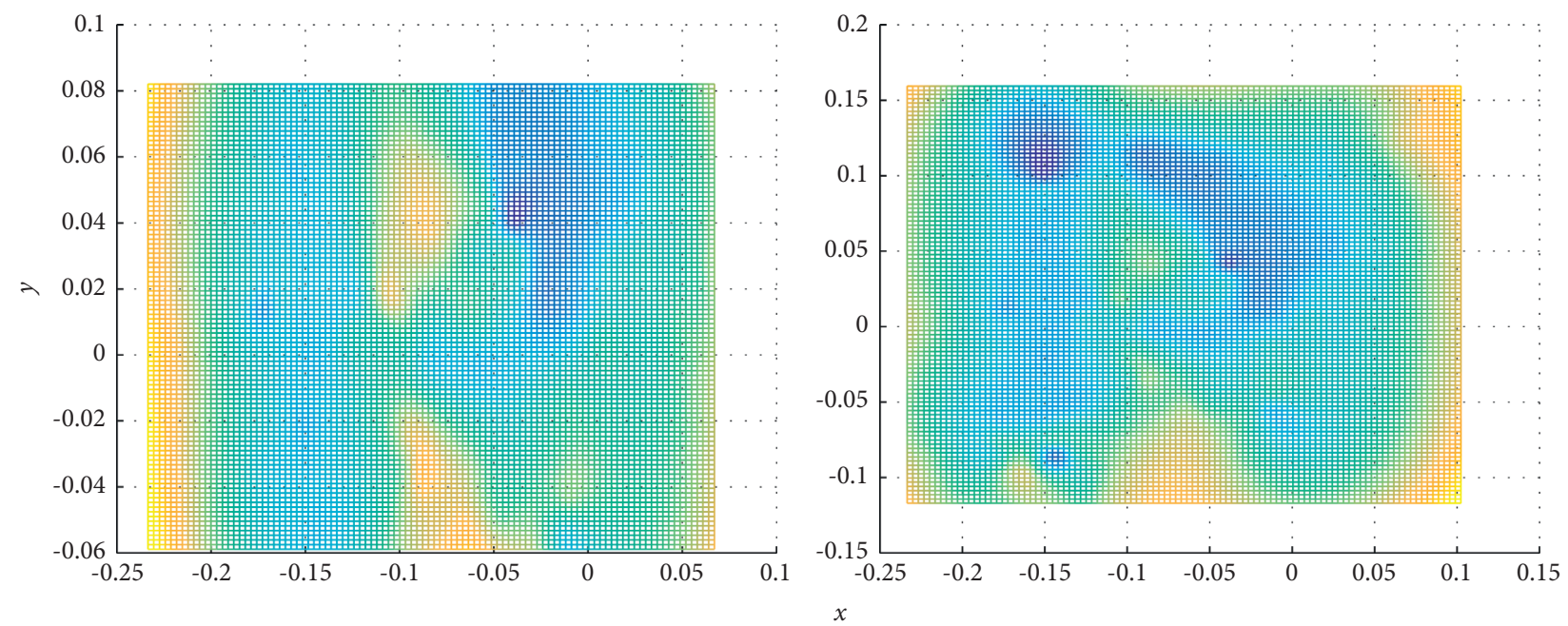

FIgURE 4: Evaluated $x$ and $y$.
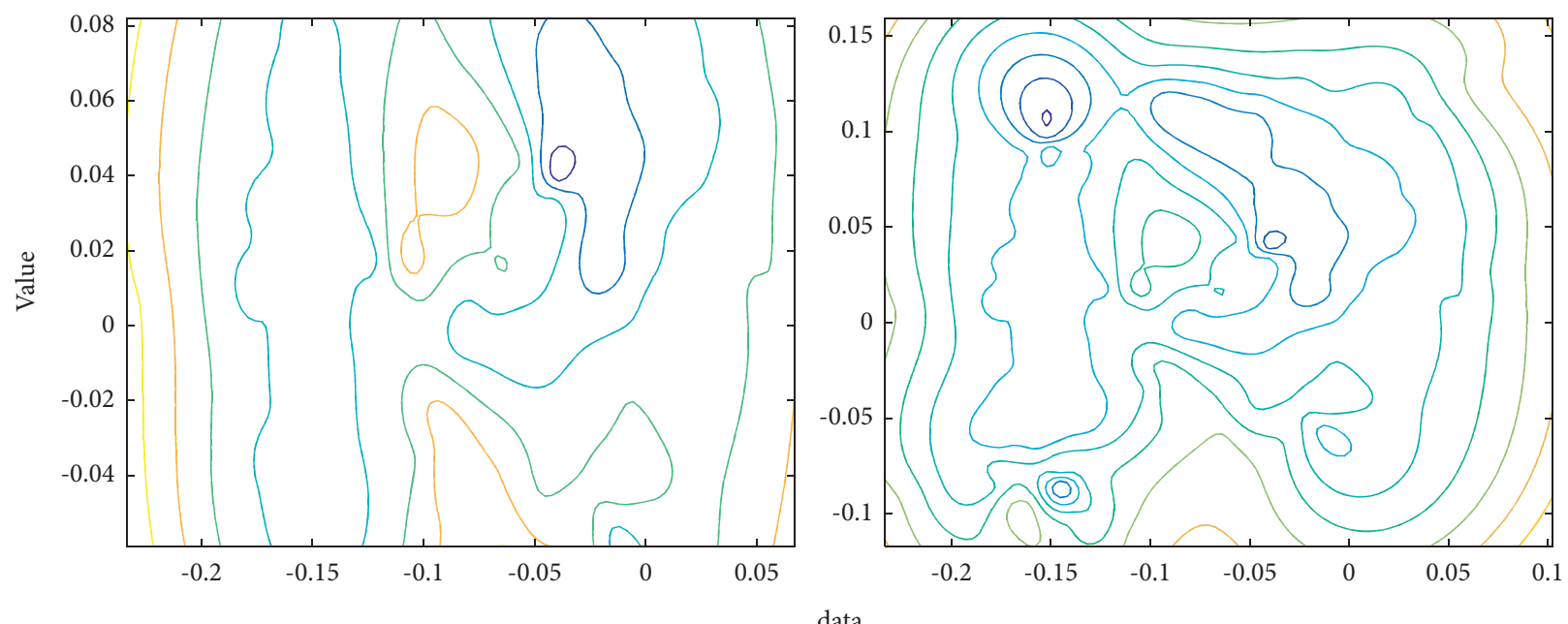

Figure 5: Predicted value.

etc. Objects represent the water channel and its spatial scene in a true three-dimensional manner; use human-computer interaction technology to dynamically set the scene expression mode to realize the scene's functions such as changes in lighting and conversion of observation angles. 3. The data management platform is the foundation of the other two parts, which centrally manages all kinds of data. This module is responsible for storing system initial data, model data, channel data, terrain data, various object data, etc.; it provides various data modification, addition, deletion, and query functions; at the same time, it provides data for the results of model calculations of the water purification system. The prediction is shown in Figure 7.

The system menu includes menu items such as "display initialization," "database initialization," "system initialization," "import model," "model save," and "exit." The "display initialization" menu item is mainly used to set the control parameters of the scene display, the "database initialization" menu item is used to complete the establishment of the database and the setting of the database parameters, and the "system initialization" menu item is mainly responsible for completing the reading of channel data and establishing the system water body. In water purification model, the "import model" menu item is used to complete the import of the established system water purification model into the system, the "model save" menu item is used to export the established model in the system to a file and save it, and the "exit" menu item is used to complete the system exit operation. The data management menu includes items such as "database setting," "river bank data management," "channel data management," "loading object management," "coordinate point management," "cross section management," and "terrain data management." The "database settings" menu item is mainly used to set the correspondence between the system and the tables in the database and the extraction of database parameters. The "river bank data management" menu item mainly maintains the spatial data information of the river bank; the "loading object management" menu item is mainly 

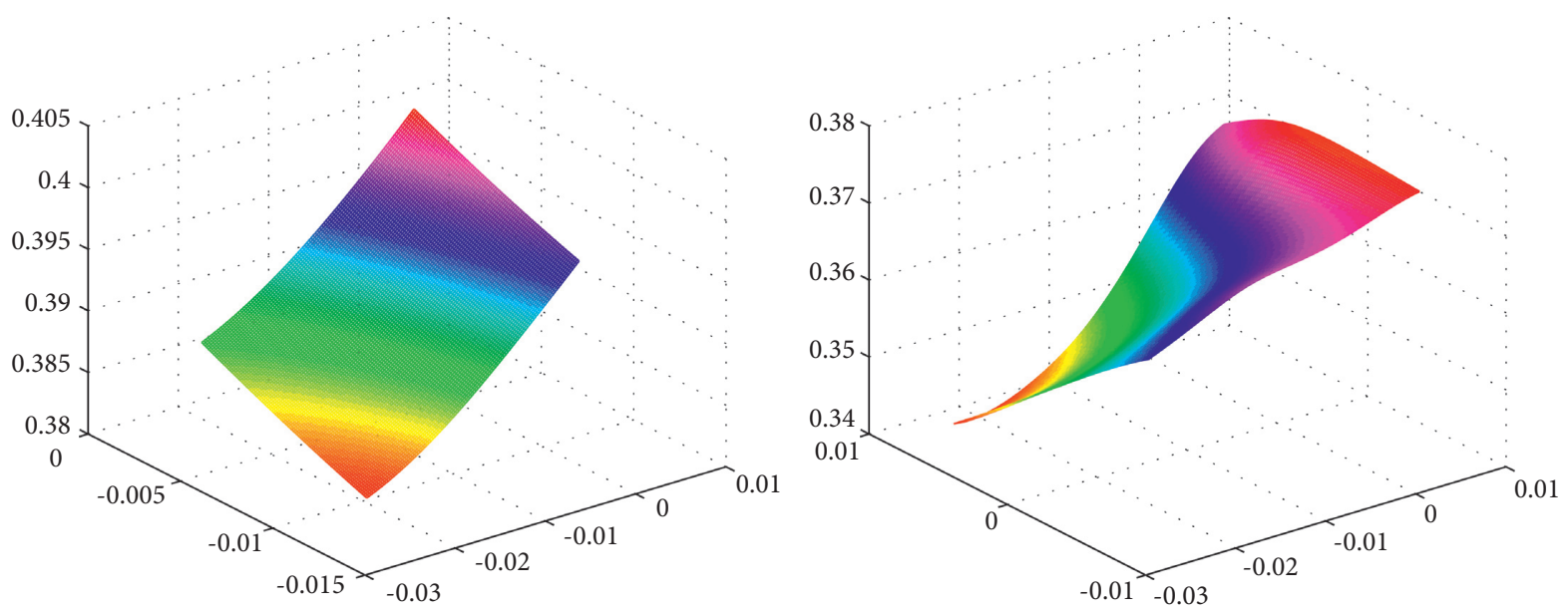

Figure 6: Prediction.

used to load 3ds Max objects into the system library; the "coordinate point management" menu item is used to complete the functions of adding, deleting, modifying, and querying all spatial coordinate points in the system; and the "cross section management" menu item is used to manage each item in the system. For the cross section data of the channel, the menu item "terrain data management" is responsible for the management of the terrain elevation data in this system. The Fourier analysis is compared in Figure 8.

The design of the terrain $3 \mathrm{D}$ visualization simulation subsystem is based on the construction of the terrain elevation model as the main axis; establishes the terrain category for management, drawing, and interpolation and other related spatial data; and designs and presents the terrain 3D visualization simulation system. 1. Realization of terrain elevation model. The terrain elevation model is based on discrete spatial elevation points, and the terrain model is created by constructing nonrepetitive triangles. The data in different position is shown in Figure 9.

The specific process is as follows. (1) Data preprocessing: The discrete spatial elevation point data, such as channel key point coordinates, ground feature key point coordinates, and actual measured elevation point coordinates, are sorted according to the spatial distribution. After abnormal points are eliminated, the maximum $X, Y, Z$ values and the minimum $X, Y, Z$ values of the display area are formed. Provide a reference for the display parameters for the simulation scene setting. (2) Spatial interpolation processing: The distance power inverse ratio method is used to interpolate the space where the known points are relatively sparse, so as to achieve a relatively uniform point density and lay the foundation for the later construction of the triangle model. (3) Construct a triangle, according to the original space point and the space coordinate value of the interpolation supplementary point, use the triangle to connect all space points, cover the entire terrain area, and form a terrain model. In the construction process, the system adopts the regular spatial interpolation method in the process of spatial interpolation and uses an interpolation radius of $5 \mathrm{~m} \times 5 \mathrm{~m}$ to perform interpolation to obtain regular gridded spatial coordinate data. These gridded spatial data are stored in the system using a two-dimensional array for three-dimensional space rendering. 2. Rendering of terrain elevation model. In the process of terrain $3 \mathrm{D}$ visualization, the regular grid data established by the terrain elevation model established above is used to complete the $3 \mathrm{D}$ simulation rendering of terrain using triangle-based surface rendering technology. The process includes the following steps. (1) Establish the terrain class according to the preprocessing results and simulation needs, constructing the destructor and the initialization of the terrain, rendering, and some texture-related functions. (2) Obtain the model, associate the model with the terrain class, instantiate the terrain class to form a terrain object, and at the same time copy and store the two-dimensional array of the elevation model to obtain the elevation data of the terrain. (3) Draw the model. According to the grid vertex data constructed by the two-dimensional array, a grid is split into two triangles, and the triangles are drawn in a counterclockwise order. After drawing the triangle patch for the entire area, the preliminary three-dimensional visualization of the terrain model is basically completed. However, in the actual process, you need to further modify and add effects (such as color settings, texture settings, light settings, and material settings) to achieve a more realistic drawing effect.

The channel 3D visualization simulation subsystem includes several aspects such as cross-sectional data management, channel data management, purification system data management and calculation, and 3D channel modeling and drawing. 1. The organization of the channel model. The channel model is based on cross-sectional information, and the channel model is constructed through the combination of two adjacent cross-sections. The specific process is as follows. (1) Data preprocessing, extracting two adjacent section data, corresponding to vertices, and forming 8 ordered vertices. (2) Construct a channel model, compose a trapezoidal or rectangular quadrangular prism based on the coordinate information of the 8 vertices, corresponding to the channel data in the database, extract various parameter information of the channel, and establish a three-dimensional channel model. 2. Drawing the channel model. In the 

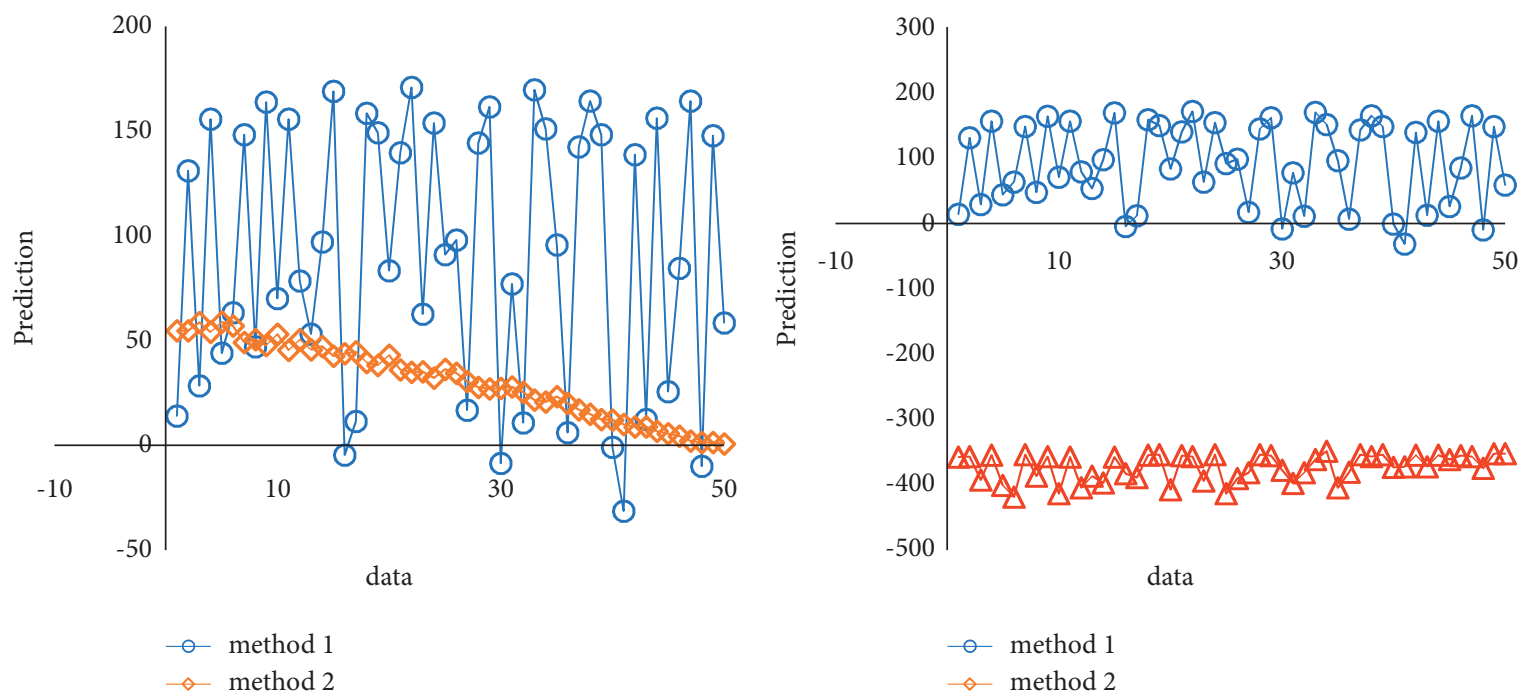

FIgure 7: The prediction.
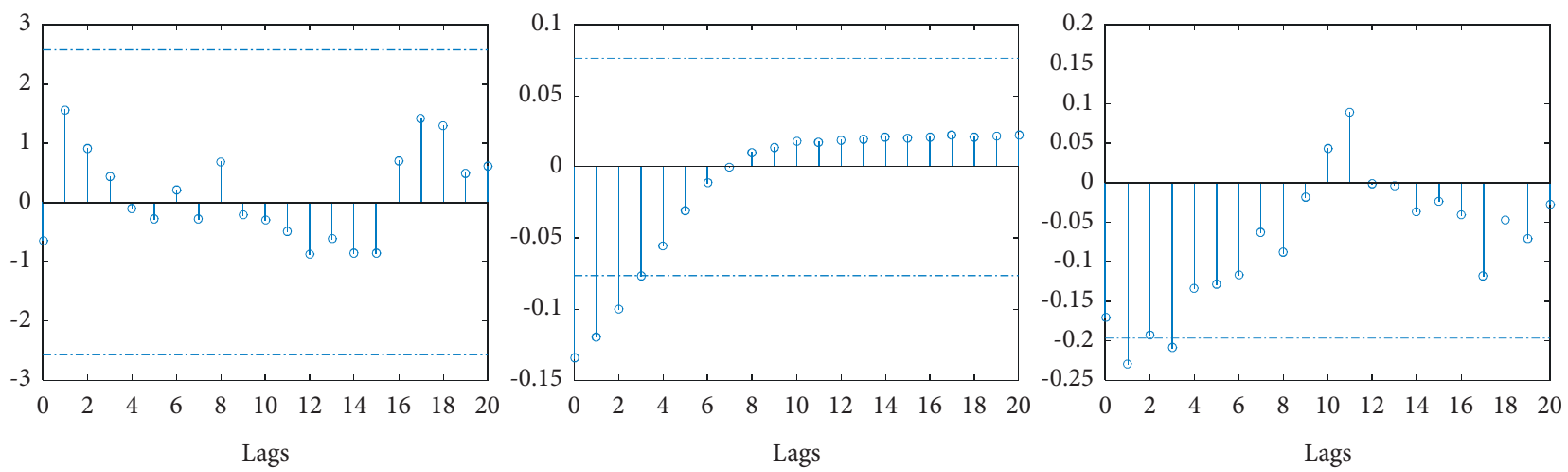

FIgURE 8: Fourier analysis.

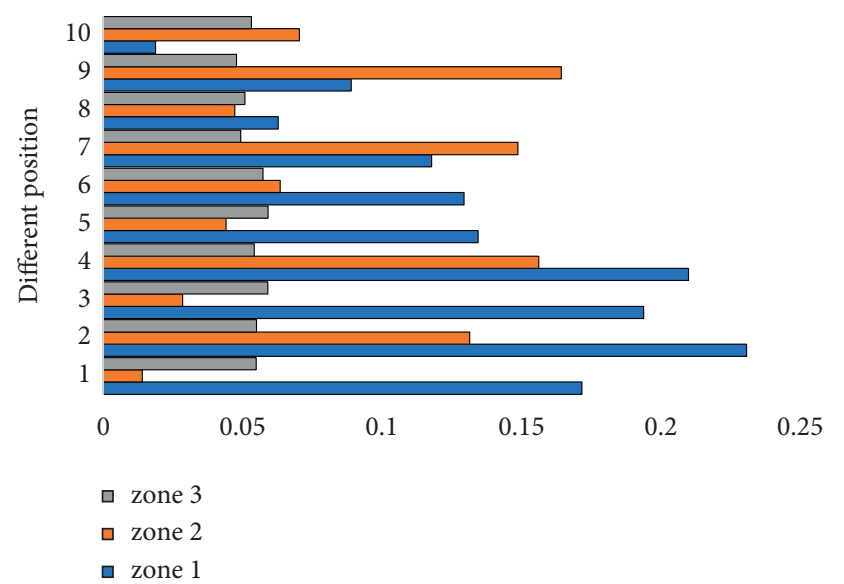

FIgURe 9: Data in different position.

process of channel three-dimensional visualization, the channel model established above is used to complete the three-dimensional drawing of each surface of the channel using quadrilateral-based surface rendering technology. The process includes the following steps. (1) Establish the channel class, and construct the channel's destructor, initialization, rendering, and some texture-related functions according to the preprocessing results and simulation needs. (2) Obtain the model, associate the model data with the channel class, instantiate the channel class to form the channel object, copy and store the channel's spatial data information and organizational structure information, and construct the left and right dams and a bottom model of the channel. (3) Draw the model. According to the coordinate information of each vertex of the channel model, draw the left and right dams and the bottom model in a quadrilateral manner, and give different texture feature maps to achieve a more realistic drawing effect, which is shown in Figure 10.

The first step is modeling of other objects in the simulation process of the $3 \mathrm{D}$ visualization scene for the water purification system. The drawn objects involve not only the main objects such as terrain and channels, but also objects such as ground objects, lawns, trees, buildings, dams, river banks, and other modified objects. The structure of these objects is relatively complex, and it is difficult to express with simple three-dimensional models. 3ds Max is an excellent 3D 


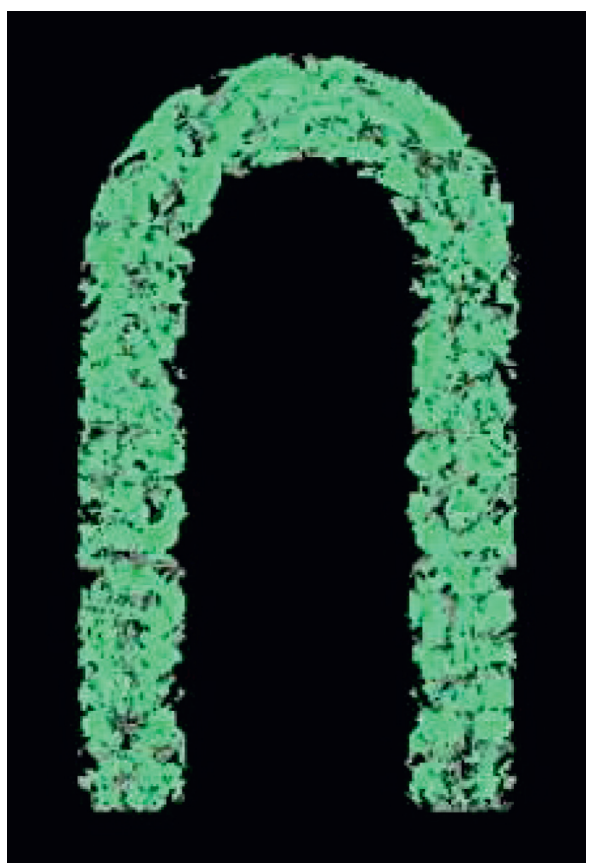

Figure 10: The effect.

modeling tool. It can easily build 3D models of various complex shapes. It is currently one of the most used static modeling tools. Therefore, $3 \mathrm{~d}$ s Max modeling tools can be used to quickly create various complex objects and attach textures, materials, and other attributes to the objects to achieve a certain rendering effect. To read and draw objects, we can use the $3 \mathrm{~d}$ s Max tool to build a variety of different three-dimensional models. The established model can be saved in the corresponding $3 \mathrm{~d}$ f file. Therefore, as long as the system can read these modeling files, it can easily convert various objects and display them further. Here, the system uses the obj file storage format as a unified interface for object reading to read objects and their textures. In this type of file, the object is decomposed into several triangular patches for storage. Therefore, after installing its storage method and reading it into the system, the object can be remodeled and drawn in the form of triangular patches. The effect is shown in Figure 9. This example is to read a $3 \mathrm{ds}$ Max rosette architecture. After the system is introduced, you can complete the translation, zoom, and rotation of the object through the control interface to achieve better observation effects.

The scene includes three parts: channel, lake surface, and terrain. The specific steps for drawing are as follows. (1) Object loading: After modeling the above objects, load these objects into the system in turn. (2) Scene modeling: Establish a view class for integrated display of $3 \mathrm{D}$ object scenes; this class inherits from CsGL GL class. Implement Init(), Resize(), and other initialization functions in this class according to the scene initialization parameters, lighting model, and projection transformation. (3) Scene drawing: Implement GL's Display() drawing function in the view class. In this function, each model is drawn in the order of object loading, and the rotation angle of the object is set in the local coordinate system according to the relevant parameters of the object. While drawing the scene in $3 \mathrm{D}$, the system adds simple human-computer interaction technology to provide technicians with a multiangle view of the $3 \mathrm{D}$ scene.

\section{Conclusion}

This paper designs a water landscape design system based on 3D image processing technology. Designers can obtain landscape design materials in the landscape design data acquisition unit and then, in the model scene design unit, realize the design based on the landscape design materials through the three-dimensional model.

(1) The design process can preprocess the 3D landscape image, remove noise information and redundant information, and use the 3D landscape image feature enhancement method to achieve 3D landscape image feature enhancement and improve the clarity of the landscape design image. The 3ds Max Material Editor is used to map the enhanced 3D landscape design to complete the design, and the designed landscape feature data will be saved in the system data statistics unit for later query.

(2) This paper also expounds the entire process of alternative simulation construction and confirms the feasibility and superiority of alternative modeling methods through multiple case comparisons. Alternative modeling methods are used as the main way to construct waterfall scenes; that is, simple models are combined with texture mapping, particle effects, and other forms to replace complex models, while ensuring the simulation effect, and the complex models are presented in the most simplified way to make the virtual scene. It is well performed in terms of authenticity and real time.

(3) The research results show that the designed system design renderings are extremely clear and of good quality; they have a high score in the water landscape design. In addition, the system application cost is low, energy consumption is low, and operation efficiency is high.

\section{Data Availability}

The dataset can be accessed upon request.

\section{Conflicts of Interest}

The author declares no conflicts of interest.

\section{Acknowledgments}

This work was supported by the Project of Anhui Province Federation of Social Sciences: "Study on the Integration Development of Urban Image of Shouxian Ancient City Environment and Calligraphy," no. 2021CX529. 


\section{References}

[1] X. Zhang, J. Yan, S. Feng, Z. Lei, D. Yi, and S. Z. Li, "Water Filling: Unsupervised People Counting via Vertical Kinect Sensor," in Proceedings of the IEEE Ninth International Conference on Advanced Video and Signal-Based Surveillance, pp. 215-220, Beijing, China, October 2012.

[2] F. Heide, W. Heidrich, M. Hullin, and G. Wetzstein, "Doppler Time-Of-Flight imaging," ACM Transactions on Graphics (ToG), vol. 34, no. 4, pp. 36-47, 2019.

[3] S. K. Dwivedi, R. Amin, V. Satyanarayana, and R. Chaudhry, "Blockchain-based secured event-information sharing protocol in internet of vehicles for smart cities," Computers \& Electrical Engineering, vol. 86, no. 1, pp. 1-9, 2020.

[4] Z. Khan and S. Amin, "Bottleneck model with heterogeneous information," Transportation Research Part B: Methodological, vol. 112, no. 1, pp. 157-190, 2018.

[5] J. M. Cairney, K. Rajan, D. Haley et al., "Mining information from atom probe data," Ultramicroscopy, vol. 159, no. 1, pp. 324-337, 2020.

[6] J. Yu and P. Lu, "Learning traffic signal phase and timing information from low-sampling rate taxi GPS trajectories," Knowledge-Based Systems, vol. 110, no. 1, pp. 275-292, 2016.

[7] K. P. Wijayaratna, V. V Dixit, L. Denant-Boemont, and S. T. Waller, "An experimental study of the Online Information Paradox: does en-route information improve road network performance?" PLoS ONE, vol. 12, no. 9, pp. 184-191, 2017.

[8] Z. Wang, H. Ren, Q. Shen, W. Sui, and X. Zhang, "Seismic performance evaluation of a steel tubular bridge pier in a fivespan continuous girder bridge system," Structures, vol. 31, no. 1, pp. 909-920, 2021.

[9] S. Nakayama and J. Takayama, "Traffic network equilibrium model for uncertain demands," in Proceedings of the 82nd Transportation Research Board Annual Meeting, Washington DC, USA, March 2021.

[10] H. Shao, W. H. K. Lam, and M. L. Tam, "A reliability-based stochastic traffic assignment model for network with multiple user classes under uncertainty in demand," Networks and Spatial Economics, vol. 6, no. 3, pp. 173-204, 2019.

[11] A. Chen, J. Kim, S. Lee, and Y Kim, "Stochastic multi-objective models for network design problem," Expert Systems with Applications, vol. 37, no. 2, pp. 1608-1619, 2020.

[12] H. Wang, W. H. K. Lam, X. Zhang, and H. Shao, "Sustainable transportation network design with stochastic demands and chance constraints," International Journal of Sustainable Transportation, vol. 9, no. 2, pp. 126-144, 2015.

[13] S.-M. Hosseininasab and S.-N. Shetab-Boushehri, "Integration of selecting and scheduling urban road construction projects as a time-dependent discrete network design problem," European Journal of Operational Research, vol. 246, no. 3, pp. 762-771, 2015.

[14] S.-M. Hosseininasab, S.-N. Shetab-Boushehri, S. R. Hejazi, and H. Karimi, "A multi-objective integrated model for selecting, scheduling, and budgeting road construction projects," European Journal of Operational Research, vol. 271, no. 1, pp. 262-277, 2018.

[15] M. Johnson, M. Schuster, Q. V. Le et al., "Google's multilingual neural machine translation system: enabling zero-shot translation," Transactions of the Association for Computational Linguistics, vol. 5, no. 1, pp. 339-351, 2017.

[16] M. D. Moreno, "Translation quality gained through the implementation of the iso en 17100:2015 and the usage of the blockchain," Babel, vol. 1, no. 2, pp. 1-9, 2020.
[17] X. Wang, X. Yu, L. Guo, F. Liu, and L. Xu, "Student performance prediction with short-term sequential campus behaviors," Information.vol. 11, no. 4, p. 101, 2020.

[18] Q. Guo, Z. Zhu, Q. Lu, D. Zhang, and W. Wu, "A dynamic emotional session generation model based on Seq2Seq and a dictionary-based attention mechanism," Applied Sciences, vol. 10, no. 6, pp. 1-10, 2020.

[19] H. Ren, Xi Mao, W. Ma, J. Wang, and L. Wang, “An EnglishChinese machine translation and evaluation method for geographical names," ISPRS International Journal of GeoInformation, vol. 9, no. 3, pp. 193-201, 2020.

[20] J. Arús-Pous, T. Blaschke, S. Ulander, J.-L. Reymond, H. Chen, and O. Engkvist, "Exploring the GDB-13 chemical space using deep generative models," Journal of Cheminformatics, vol. 11, no. 1, pp. 20-29, 2019.

[21] T. Moon, T. In Ahn, and J. E. Son, "Long short-term memory for a model-free estimation of macronutrient ion concentrations of root-zone in closed-loop soilless cultures," Plant Methods, vol. 15, no. 1, pp. 1-12, 2019.

[22] N. Pourdamghani and K. Knight, "Neighbors helping the poor: improving low-resource machine translation using related languages," Machine Translation, vol. 33, no. 3, pp. 239-258, 2019.

[23] L. Bote-Curiel, S. Muñoz-Romero, A. Gerrero-Curieses, and J. L Rojo-Álvarez, "Deep learning and big data in healthcare: a double review for critical beginners," Applied Sciences, vol. 9, no. 11, pp. 1-11, 2019.

[24] J. Zhang and T. Matsumoto, "Corpus augmentation for neural machine translation with Chinese-Japanese parallel corpora," Applied Sciences, vol. 9, no. 10, pp. 1-12, 2019.

[25] Y. Chen, Y. Ma, X. Mao, and Q. Li, "Multi-task learning for abstractive and extractive summarization," Data Science and Engineering, vol. 4, no. 1, pp. 14-23, 2019.

[26] Pu. Zhou and Z. Jiang, "Self-organizing map neural network (SOM) downscaling method to simulate daily precipitation in the Yangtze and Huaihe River Basin," Climatic and Environmental Research, vol. 21, no. 5, pp. 512-524, 2016.

[27] X. Xiao, "Analysis on the employment psychological problems and adjustment of retired athletes in the process of career transformation," Modern Vocational Education, vol. 5, no. 12, pp. 216-217, 2018.

[28] S. Sahoo and M. K. Jha, "Pattern recognition in lithology classification: modeling using neural networks, self-organizing maps and genetic algorithms," Hydrogeology Journal, vol. 25, no. 2, pp. 311-330, 2016.

[29] Y. Zhou and B. Yang, "Sports video athlete detection using convolutional neural network," Journal of Natural Science of Xiangtan University, vol. 39, no. 1, pp. 95-98, 2017.

[30] J. Pang, "Research on the evaluation model of sports training adaptation based on self-organizing neural network," Journal of Nanjing Institute of Physical Education, vol. 16, no. 1, pp. 74-77, 2017.

[31] G. Querzola, C. Lovati, C. Mariani, and L. Pantoni, "A semiquantitative sport-specific assessment of recurrent traumatic brain injury: the TraQ questionnaire and its application in American football," Neurological Sciences, vol. 40, no. 9, pp. 1909-1915, 2019.

[32] J. Wang, X. Luo, and H. Yan, "Correlation analysis between injuries and functional movement screening for athletes of the National Shooting Team," Journal of Capital Institute of Physical Education, vol. 5, no. 4, pp. 352-355, 2016.

[33] G. Ma, "Research on the design of juvenile football players' sports injury prediction model," Automation Technology and Application, vol. 277, no. 7, pp. 141-144, 2018. 